\title{
BMJ Open The KOMPACT-P study: Knee Osteoarthritis Management with Physiotherapy informed by Acceptance and Commitment Therapy-Pilot study protocol
}

\author{
Marie K March (D) , ${ }^{1,2}$ Alison Harmer, ${ }^{2}$ Emma Godfrey, ${ }^{3,4}$ Shruti Venkatesh, ${ }^{5}$ \\ Bijoy Thomas, ${ }^{6}$ Sarah Dennis ${ }^{2,7,8}$
}

To cite: March MK, Harmer A, Godfrey E, et al. The KOMPACT-P study: Knee Osteoarthritis Management with Physiotherapy informed by Acceptance and Commitment Therapy—Pilot study protocol. BMJ Open 2020;10:e032675. doi:10.1136/ bmjopen-2019-032675

- Prepublication history and additional material for this paper are available online. To view these files, please visit the journal online (http://dx.doi. org/10.1136/bmjopen-2019032675).

Received 17 July 2019 Revised 12 February 2020 Accepted 21 April 2020

Check for updates

(c) Author(s) (or their employer(s)) 2020. Re-use permitted under CC BY-NC. No commercial re-use. See rights and permissions. Published by BMJ.

For numbered affiliations see end of article.

Correspondence to

Marie K March;

Marie.March@health.nsw.gov.au

\section{ABSTRACT}

Introduction Incidence of total knee arthroplasty (TKA) is projected to rise $276 \%$ in 2030 , and psychological distress affects up to $42 \%$ of people with knee osteoarthritis undergoing TKA, with demonstrated detrimental effects on postoperative outcomes. Few studies have assessed psychological treatment in people awaiting TKA, and these have been psychologist-delivered treatments. No evidence exists regarding psychologically-informed interventions delivered by health professionals currently embedded in TKA clinical pathways. The primary aim of this pilot study is to explore the safety, acceptability and feasibility of

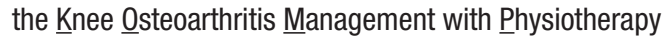
informed by Acceptance and Commitment Therapy (KOMPACT) approach in people awaiting TKA.

Methods and analysis 51 community-dwelling adults scheduled for a primary TKA at two hospitals will be recruited to this pilot, mixed-methods, prospective randomised controlled trial with assessor blinding. Participants will be randomised in a 1:2 ratio to either usual care (education class) or usual care plus KOMPACT ( 2 hours 20 min of preoperative physiotherapy informed by Acceptance and Commitment Therapy). Our primary outcome measures are safety (length of stay, complications and psychological health after KOMPACT), acceptability (treatment credibility and qualitative data) and feasibility (recruitment, retention and intervention fidelity) of the KOMPACT approach. Secondary outcomes include health service outcomes, patient-reported physical and psychological outcomes, and physical performance measures. Quantitative data collection was conducted at baseline, 1-2 weeks before TKA, 6 weeks after TKA and 6 months after TKA. Qualitative data collection is 1-2 weeks before TKA. Data analysis will take a quantitative-led approach with triangulation after thematic analysis of the qualitative data.

Ethics and dissemination This study has full ethics approval (HREC/18/WMEAD/440). Results from this study will be published in peer-reviewed journals and presented at local and international conferences.

Trial registration number Australia New Zealand Clinical Trials Registry (ACTRN12618001867280p).

\section{Strengths and limitations of this study}

- The use of mixed methods at the early stages of intervention development allows refinement of the

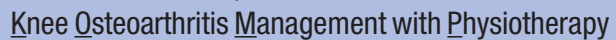
informed by Acceptance and Commitment Therapy (KOMPACT) intervention before full-scale testing, limiting research waste for future trials.

- Time and funding constraints have led to a 2:1 randomisation design in order to gather sufficient qualitative and quantitative data for the KOMPACT intervention.

- This study demonstrates a robust method of assessing intervention fidelity.

\section{INTRODUCTION}

Total knee arthroplasty (TKA) procedures impose a high-cost burden on health services worldwide. ${ }^{1}$ In Australia, over 63000 knee arthroplasty procedures were performed in 2017, costing approximately \$A20 000 per procedure. ${ }^{23}$ Incidence of TKA is projected to rise $276 \%$ from 2013 to 2030, with projected costs to increase by $\$$ A521 million over the same time period, ${ }^{4}$ so optimising health outcomes following TKA is important to ensure that costs are restrained and TKA procedures remain high value.

Preoperative medical, surgical and sociodemographic factors influence postoperative TKA outcomes, but recent evidence suggests that preoperative psychological distress independently predicts longer hospital length of stay, higher pain levels and higher dissatisfaction at 6 months following TKA. ${ }^{5-7}$ However, few clinicians assess or treat psychological health before TKA, despite up to $42 \%$ of preoperative patients reporting psychological distress. $^{8}$ 
Preoperative interventions to improve outcomes after TKA have mixed effects. ${ }^{9}{ }^{10}$ Most of these interventions are standardised exercise and/or education and delivered by physiotherapists, with no psychological interventions used. Only two pilot studies have used robust psychological interventions before TKA, with promising results. ${ }^{11}{ }^{12}$ These studies added mental health professionals to existing orthopaedic teams to provide psychological interventions, but this is difficult to implement equitably for health services worldwide, given the high volume of patients who may benefit. Equipping professionals already embedded in orthopaedic teams, such as physiotherapists, to provide psychologically-informed preoperative intervention may be a feasible and effective alternative. Psychologically-informed physiotherapy is a growing area of clinical practice and research in the management of chronic musculoskeletal pain and osteoarthritis, ${ }^{13} 14$ which has been well received by patients and physiotherapists. ${ }^{15}$

We have developed a physiotherapist-delivered, psychologically-informed intervention based on Acceptance and Commitment Therapy (ACT). ACT is more effective than traditional cognitive-behavioural therapy for older people experiencing chronic pain ${ }^{16}$ and is focused on developing psychological flexibility to promote values-based behaviour change. Psychological flexibility is a transdiagnostic concept, and lack of psychological flexibility is associated with functional impairment in people experiencing chronic pain. ${ }^{17}$ This is achieved by improving the key domains of openness, awareness and engagement. Our intervention, named KOMPACT

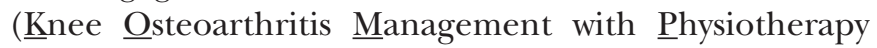
informed by Acceptance and Commitment Therapy), is focused on the engagement domain, with identification of core values, and subsequent emotionally relevant behavioural goal setting as explicit inclusions from an ACT perspective. This domain intersects well with physiotherapy clinical practice, evidence-based guidelines for knee osteoarthritis management ${ }^{1819}$ and evidence that physiotherapists can successfully implement ACT with people experiencing chronic pain. ${ }^{20}{ }^{21}$ The domains of openness and awareness will be more implicit to ensure that KOMPACT is feasible and relevant for physiotherapists and tesheir clients. ACT also specifies an equal, respectful and empathetic therapeutic stance with a mandate not to challenge beliefs but to accept them. ${ }^{22} \mathrm{~A}$ strong therapeutic stance is important as it is associated with perceived improvement in chronic musculoskeletal pain. ${ }^{23} 24$

This pilot study is testing the KOMPACT intervention in people awaiting TKA. The primary aim of this pilot study is to evaluate the safety, acceptability and feasibility of the KOMPACT approach among patients awaiting TKA according to success criteria determined a priori. This approach consists of four physiotherapy sessions over 2 months before and after TKA in a public hospital setting, including psychologically-informed goal setting, lower limb strength training and physical activity coaching, in addition to usual care. The secondary aim is to collect outcome data on effect size and variation for a range of variables (health service, psychological health, patient-reported outcomes, quality of life and physical performance measures) that will inform the design and sample size for a future randomised controlled trial (RCT).

\section{METHODS AND ANALYSIS \\ Design}

This is a mixed-methods, parallel-group, pilot RCT with recruitment at two hospital sites in Sydney, Australia. Participants will be randomised into one of two groups: usual care or KOMPACT plus usual care.

\section{Sample size estimation}

We aim to recruit 51 participants, which is adequate to enable randomisation with stratification for sex, site and psychological state. Stratification will ensure sufficient qualitative data regarding KOMPACT acceptability and no significant differences between groups due to three factors: anticipated $70 \%$ female participants ${ }^{2}$; current local procedures that determine hospital site (those with higher anaesthetic risk are operated on at one site only), and anticipation of a 30\% psychological distress rate. ${ }^{5}$ Considering most physiotherapy interventions have a small effect size of 0.2 and a two-sided significance of $\mathrm{p}=0.05$, our sample size met recommendations by Whitehead and colleagues for external pilot randomised trials. ${ }^{25}$ This sample size was also considered feasible for research conducted within a health service and is consistent with available time and funding for recruitment.

\section{Participants}

We will recruit 51 sequential participants who are awaiting primary unilateral TKA, drawn from two hospital sites that performed a combined total of approximately 400 arthroplasty procedures in 2018 (Blacktown Hospital and Mount Druitt Hospital).

Potential participants will be considered eligible for inclusion in this study if they are:

- Over 18 years of age.

- Accepted for the surgical waiting list for an elective primary unilateral TKA for osteoarthritis at participating hospitals.

- Attending preadmission clinic appointments at the same site as their surgery.

Potential participants will be excluded from the study if they:

- Have cognitive impairment preoperatively on screening using the Graphical Mini-Cog. ${ }^{26}$

- Reside outside the Western Sydney Local Health District.

- Are scheduled for revision, unicompartmental or bilateral TKA.

- Have further orthopaedic surgery planned in the next 6 months. 
- Report acute psychological distress at the time of screening.

- Report current use of illicit substances or alcohol intake $>14$ units per week.

People who are interested and eligible for the study but have insufficient English language skills to engage with KOMPACT will be referred to a government-funded telephone coaching service available in different languages (Get Healthy https://www.gethealthynsw.com.au) to support lifestyle-related behavioural goal setting.

\section{Recruitment method and screening procedures}

Potential participants awaiting TKA will be scheduled by administration staff to attend the usual care preadmission class approximately 2 months before surgery as per usual clinical care. On attendance, all potentially eligible participants will be provided with information regarding study participation by a study investigator, given the opportunity to ask questions and, if agreeable, will be invited to consent to screening for the study. If the participant remains eligible after screening, then they will be asked to provide written informed consent. Once consented, and prior to treatment allocation, baseline measures will be collected by an allied health student or staff member trained by the lead author in the requisite standardised assessment procedures, who is blinded to allocation. Recruitment will commence February 2020 and will be completed by December 2020, however, this may be delayed due to the COVID-19 pandemic.

\section{Randomisation}

Participants will be randomised in a 1:2 allocation to either usual care $(n=17)$ or the KOMPACT treatment group $(\mathrm{n}=34)$.

Once consent and baseline assessments are complete, participants will be randomised to either the usual care group or KOMPACT treatment group. This will occur via a computerised process of randomisation by minimisation, with algorithms constructed by an independent consulting statistician. An offsite investigator will be contacted when a random allocation of participants is required. Randomisation will be stratified for three binary variables: sex, hospital site and psychological health (if the score in any domain of the Depression, Anxiety and Stress Score-21 (DASS-21) above validated 'normal' threshold, participant classified as 'distressed' $;{ }^{27}$ note the DASS-21 is valid in older people with chronic disease ${ }^{28}$ ). Unequal randomisation favouring the KOMPACT treatment group will generate more quantitative and qualitative data regarding this intervention and allow for any learning effects from the treating physiotherapist using KOMPACT.

\section{Intervention}

Usual care

The usual care group (and accompanying support people) will attend a 1-hour education class at the physiotherapy department at 2 months before surgery. This class and the supporting education manual will describe the usual clinical pathway for TKA at these sites, the role of health professionals involved with TKA care, address expectations of recovery, demonstrate two exercises to address knee range of motion, outline follow-up plans after discharge from hospital and encourage independent goal setting for return to usual activities. They will receive usual postoperative physiotherapy on the ward at least once daily to improve mobility and knee range of motion.

\section{KOMPACT treatment group}

The treatment group will receive four one-to-one KOMPACT sessions with the KOMPACT physiotherapist (2 hours $20 \mathrm{~min}$ total duration), in addition to usual care. The initial appointment will occur within 2 weeks of recruitment, with follow-up sessions at 3 weeks and 6 weeks afterwards. The fourth appointment will occur during the inpatient stay in the orthopaedic ward after TKA. This dosage parallels Godfrey and colleagues' intervention of physiotherapy informed by ACT for chronic low back pain. ${ }^{20}$ Box 1 describes the KOMPACT intervention at each session in detail, and table 1 provides some examples of how ACT is applied in KOMPACT. The therapeutic stance adopted by KOMPACT will be integrated into the application of psychological skills and exercisebased interventions.

The use of behaviour change techniques in physiotherapy is a growing area of research, as many physiotherapy interventions depend on patients independently implementing prescribed strategies outside of the supervised clinic. The Behaviour Change Taxonomy $(\mathrm{BCT})^{29}$ itemises behaviour change techniques used to standardise identification and reporting of techniques used, for health professionals within and outside the psychological sciences. Both usual care and KOMPACT interventions were mapped to the BCT (online supplementary appendix 1) by the lead author, with independent checking by two authors (EG, SD). The lead author undertook online training in BCT mapping as prescribed by the authors of the BCT.

\section{Therapist training}

The single physiotherapist delivering KOMPACT treatment will undergo a 16-hour online course "ACT for Beginners, ${ }^{30}$ with an additional 1 day of trial-specific KOMPACT training conducted by a clinical psychologist and an experienced physiotherapist familiar with psychologically-informed physiotherapy and KOMPACT, complemented by a therapist training manual. The KOMPACT physiotherapist has over 10 years' experience in orthopaedic and musculoskeletal physiotherapy and is familiar with the ACT therapeutic stance. The KOMPACT physiotherapist will participate in monthly reflective supervision from a clinical psychologist (SV) in person or via video-conferencing to improve KOMPACT intervention fidelity and ensure psychological safety for the KOMPACT physiotherapist. 


\section{Box 1 KOMPACT intervention}

\section{Session 1: $60 \mathrm{~min}$; before TKA}

- Musculoskeletal physiotherapy subjective and objective assessment, with reference to patient-reported outcomes.

- Feedback given in the ACT style linking initial assessment findings, participant experience and expectations of TKA.

- One metaphor* used to elicit behaviour change.

- One ACT skill† taught to improve psychological flexibility.

- Identification of core values.

- SMARTER goal setting for desired physical activity behaviour.

- Action plan and relapse prevention strategies identified and documented for SMARTER goal.

- Behavioural contract with signature, handshake and commitment to discuss with one person goals.

- Prescription of one lower limb strength exercise linked to identified impairments and functional goals at 8-10 Repetition Maximum.

- Action plan documented for strength exercise.

- Initial discussion of progression principles.

- Patient manual issued.

\section{Session 2: $30 \mathrm{~min}$; before TKA}

- Positive review of progress using ACT therapeutic stance.

- Normalised setbacks and encouraged attempts.

- Review of SMARTER goal linked to desired physical activity behaviour

- Adjust the action plan for the SMARTER goal if required.

- Review of strength exercise.

- Progression of strength exercise with a collaborative approach.

- Generalisation of psychological flexibility to other areas of life.

- One (different) ACT skill taught to improve psychological flexibility.

- Optional: use of ACT metaphor to elicit behaviour change if required.

\section{Session 3: $30 \mathrm{~min}$; before TKA}

- Positive review of progress using ACT therapeutic stance.

- Normalised setbacks and encouraged attempts.

- Review of SMARTER goal linked to desired physical activity behaviour.

- Adjust the action plan for the SMARTER goal if required.

- Review of strength exercise.

- Progression of strength exercise with a patient-led approach

- Review of use of ACT skills to improve psychological flexibility in other areas of life.

- Positive sign off.

\section{Session 4: $20 \mathrm{~min}$; after TKA}

- Positive review of inpatient progress.

- Identification of difficult tasks as inpatient.

- Apply ACT skill (previously learnt) in this context.

- Normalised setbacks and encouraged attempts to engage in desired behaviours.

- Positive closure regarding progress and future follow-up.

- Optional: refer to inpatient mental health professionals if required.

*Metaphors are stories that connect with the patient's experience and allow them to reframe their perspective.

†ACT skills in this study are exercises that promote psychological flexibility and are usually focused on the 'openness' and 'awareness' domains of ACT. ACT, Acceptance and Commitment Therapy; KOMPACT, Knee ㅇsteoarthritis Management with Physiotherapy informed by Acceptance and $\underline{\text { Commitment }}$ Therapy; SMARTER, Specific Measurable Achievable Realistic Timely

Emotionally Relevant; TKA, total knee arthroplasty.

\section{Intervention fidelity}

Intervention fidelity will be assessed with an a priori checklist tailored to KOMPACT, assessing adherence and competence in key components (online supplementary appendix 2). All treatment sessions will be audiorecorded and the supervising clinical psychologist will listen to at least a $10 \%$ sample for supervision and fidelity purposes. The nature of the study renders both therapist and participant blinding impossible. Usual care will be standardised with the provision of presentation slides, notes and manuals, and the KOMPACT physiotherapist will not provide usual care for any participants. Usual care fidelity will not be formally assessed as participants not enrolled in the study will also be present and unable to give consent.

\section{Primary outcomes}

All outcomes will be assessed by an assessor blinded to treatment group allocation. The primary outcomes for this pilot RCT are safety, acceptability and feasibility. Safety will be assessed using DASS-21 data after preoperative KOMPACT treatment, postoperative length of stay, and complications. Acceptability will be determined using treatment credibility scores developed by Borkovec and Nau tailored for KOMPACT, ${ }^{31}$ in addition to qualitative data. Feasibility will be determined using recruitment and retention rates, recruitment time, attendance rates and intervention fidelity. Specific criteria for success are stated in the online supplementary appendix 3.

\section{Secondary outcomes}

Health services measures

1. Relevant sociodemographic, anthropometric, medical history and attendance data will be ascertained from medical records.

2. Acute hospital length of stay in calendar days, incidence of complications and inpatient rehabilitation use will be ascertained from electronic medical records.

3. Physiotherapy inpatient occasions of service will be ascertained from standardised data collection procedures in NSW Health.

4. Patient satisfaction with TKA will be measured with a single question after TKA and explored during semistructured interviews.

\section{Psychological health measures}

1. Brief Resilience Scale ${ }^{32}$ : a six-item self-administered scale with responses from 'strongly agree' to 'strongly disagree', with three items reverse scored. The final score is calculated as the mean of the responses, with scores $<3.00$ considered low resilience.

2. Committed Action Questionnaire- $8^{33}$ : the eight-item version has responses ranging from 'never true' (score of 0 ) to 'always true' (score of 6), with scores summed to give a total value. Higher scores indicate higher psychological flexibility. 
Table 1 ACT domains and strategies in KOMPACT

\section{Domain KOMPACT strategy to address domain}

\section{Openness}

Patient response: 'I am open to the experience of pain without resisting it'

Willingness to experience undesirable thoughts and feelings such as pain without needing to challenge or change them

\section{Example ACT Metaphor: 'Ball and chain'}

Having chronic pain can be like having a heavy ball and chain attached to your leg. It can drag you down, pin you to the spot and stop you from going the way you want to go. Your instinct is to pull against the ball and chain, trying to leave it behind so that you can get on with things

What if you stopped struggling with the ball? Instead of pushing against the ball and chain, trying to leave it behind, what if you brought it with you?

\section{Awareness}

Patient response: 'I am more than my pain experience'

Separation of undesirable experience of pain from Example ACT Skill of mindfulness: 'Notice five things' own identity

Ensure connection to present moment experience, 1. Pause. incorporating all sensory experiences, instead of past or future experiences such as pain.

2. Look around and notice five objects you can see... (wait at least 10 seconds).

3. Listen carefully and notice five sounds you can hear... (wait at least 10 seconds).

4. Notice five things you can feel on the surface of your skin... (wait at least 10 seconds).

\section{And, stop.}

6. What happens during this exercise for you?

\section{Engagement}

Patient response: 'Being active is important to me, and I may have pain doing it.'

\section{Identify desired values and persistently take} action towards these values, alongside difficult experiences such as pain

\section{Values-based SMARTER goal setting}

Values: patient $X$ values friendships, hospitality, honesty

SMARTER goal: patient $X$ will complete three sets of knee squats to fatigue on Tuesdays and Thursdays to improve her knee strength so that she can continue walking with her friends on Mondays and Wednesdays and maintain friendships.

ACT, Acceptance and Commitment Therapy; KOMPACT, Knee Osteoarthritis Management with Physiotherapy informed by Acceptance and Commitment Therapy; SMARTER, Specific Measurable Achievable Realistic Timely Emotionally Relevant.

3. Chronic Pain Acceptance Questionnaire-8 $8^{34}$ : this eightitem questionnaire measures two aspects of psychological flexibility-activity engagement and pain willingness. Higher scores indicate higher psychological flexibility.

4. Pain Self-efficacy Questionnaire- $2^{35}$ : this two-item scale asks respondents to rate their confidence on a visual analogue scale from 0 ('not at all confident') to 6 ('completely confident'). Total scores of $\leq 5$ indicate low pain self-efficacy needing intervention.

5. DASS-21 ${ }^{27}$ : this 21 -item modified version asks participants to rate how frequently each item applied to them, on a 4-point Likert scale from 'never' (score of 0) to 'almost always' (score of 3). Summed scores for each component (depression, anxiety and stress) are tallied, and higher scores indicate higher distress. Recommended thresholds are defined for normal, mild, moderate, severe and extremely severe distress in each domain.
Patient-reported measures

1. Oxford Knee Score ${ }^{36}$ : this self-report measure of pain and function has been validated for participants after TKA, with low scores indicating high pain and disability. The minimum clinically important difference after TKA is 4 points.

2. Numerical Pain Rating Scale ${ }^{37}$ : administered in written or verbal forms, this will assess the worst pain in the past 24 hours, where higher scores indicate worse pain.

3. International Physical Activity Questionnaire for the Elderly: validated for those over 65 years, ${ }^{38}$ this measures self-reported frequency and duration of sitting behaviour, walking, moderate activity and vigorous activity.

\section{Quality of life measures}

1. EuroQoL-5D-5L ${ }^{39}$ : comprising two sections, the first assessing self-reported health-related quality of life in five domains, with higher scores representative of the 
Table 2 Outcome measures

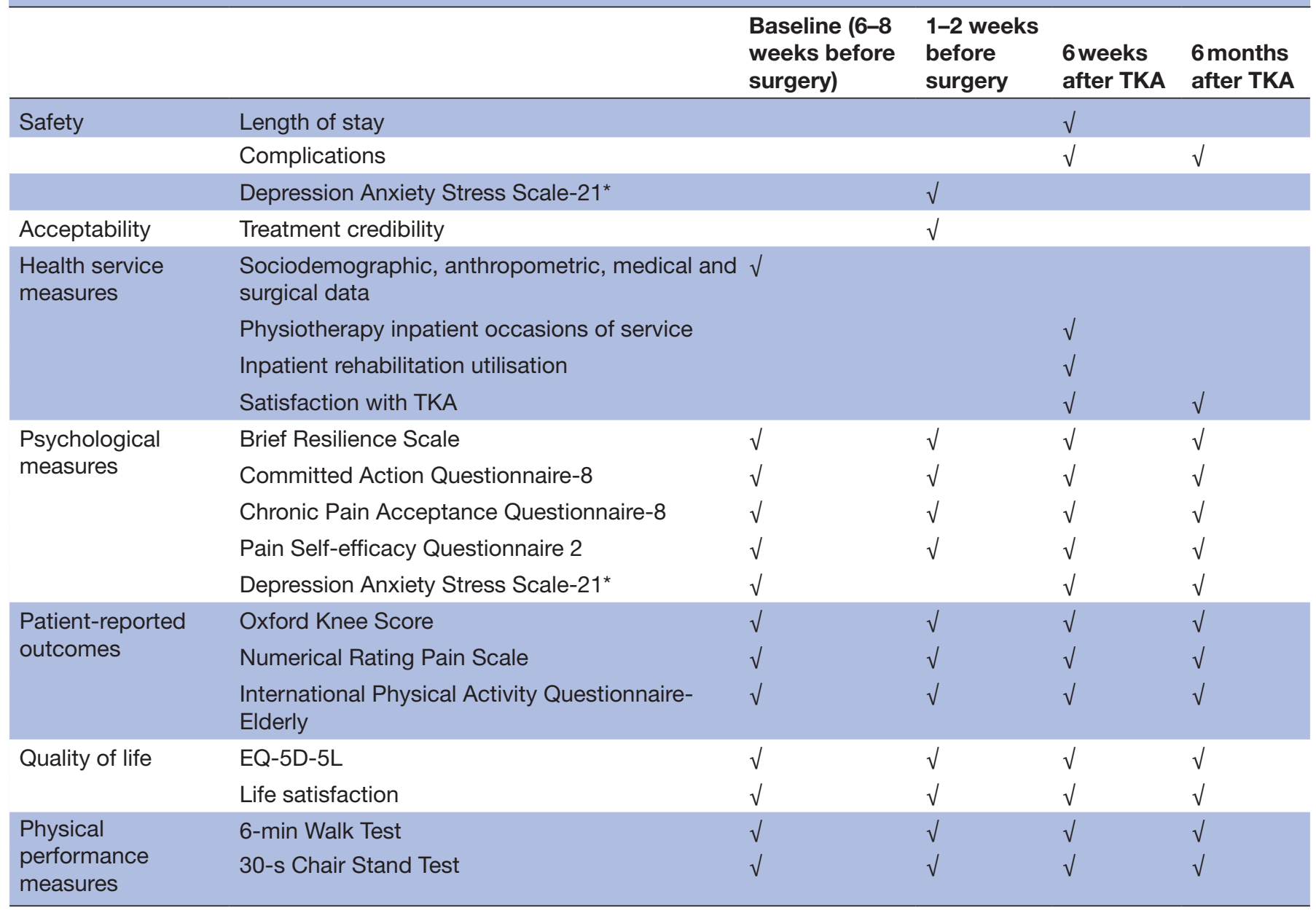

*Depression, Anxiety and Stress Scale-21 scores immediately after KOMPACT will be used to assess safety as a primary outcome. It is included here for completeness, and to document how this outcome is measured at subsequent timepoints.

EQ-5D-5L, EuroQoL-5D-5L; KOMPACT, Knee Osteoarthritis Management with Physiotherapy informed by Acceptance and Commitment Therapy; TKA, total knee arthroplasty.

worse quality of life. The second section is a visual analogue scale where subjects rate their health today from 0 to 100 , where high scores indicate 'best health'.

2. Life satisfaction will be assessed by a single question using an 11-point Numerical Rating Scale, where high scores indicate high satisfaction.

Physical performance measures

1. Six-minute walk test: this standardised walking tolerance test measures maximum walking distance in 6 minutes, with self-initiated rests as desired. It is valid, reliable and recommended for those with knee osteoarthritis. ${ }^{4041}$

2. 30 second chair stand test: this is a validated proxy measure of leg strength, ${ }^{42}$ with participants required to stand up from a standardised chair as many times as they can over 30 seconds, with a number of repetitions recorded.

A summary of outcome measures and the time of collection is provided in table 2 .

\section{Data analysis}

An independent biostatistician assisted in developing the data analysis plan and randomisation methods and will be involved in data analysis. Quantitative data will be described with measures of central tendency and variation appropriate to their distribution, allowing estimation of effect size, sample size and selection of outcome measures for a future fully powered trial. Qualitative data will be acquired by purposive sampling and oneto-one, semistructured interviewing of $\geq 10$ participants in the treatment group. Purposive sampling will occur considering sociodemographic variables, level of psychological distress and KOMPACT completion. Informal checking during the interview process will occur, and after transcription of interviews verbatim, the thematic analysis will be performed, with two researchers involved in developing a framework within which the data will be interpreted, and major themes described. ${ }^{43}$ Formal member checking will not occur to minimise patient burden during the acute recovery from TKA and to avoid 
confounding by postoperative experiences. Our mixedmethods approach will be a triangulation approach, as qualitative data will inform and enrich the interpretation of the quantitative data and inform a future efficacy trial of KOMPACT.

Quantitative data will be checked on collection to ensure completeness, with prompts for completion if required. Data will be checked for correctness during data analysis, and if required, missing values will be carried forward from previous assessments. Imputation will not occur from preoperative to postoperative variables.

\section{Patient and public involvement}

No patients or members of the general public were involved in the design of this study. However, qualitative study data will allow the research team to incorporate participant feedback for any future studies using KOMPACT.

\section{ETHICS AND DISSEMINATION}

The study design was conducted in accordance with the Standard Protocol Items: Recommendations for Interventional Trials (SPIRIT) guidelines for trial protocols. ${ }^{44}$ The KOMPACT intervention will be reported in accordance with the Template for Intervention Description and Replication (TIDieR) guidelines for complex interventions. ${ }^{45}$ Results will be reported in accordance with the Consolidated Standards of Reporting Trials guidelines for pilot and feasibility RCTs. ${ }^{46}$ Western Sydney Local Health District Human Research Ethics Committee has provided ethical approval for this study (HREC/18/ WMEAD/440) and annual progress reports will be provided to this committee. Participants will be asked to provide voluntary written consent before enrolment, with collaboration from support persons as they wish. Opportunities to clarify what participation involves, potential risks and benefits will be provided by a study investigator, and participants are free to withdraw at any time, with no subsequent data collected. Willing potential participants whose English language skills limit participation will be referred to an appropriate service delivered in their preferred language. This study is exploring a low risk intervention with increased monitoring compared with usual care. If potential participants are experiencing acute psychological distress, then referral pathways exist for these people to access urgent specialist mental health care at no cost. The results of this study will be disseminated via peer-reviewed journals and local and international conferences.

\section{Data protection, storage and dissemination}

All data will be collected electronically and deidentified. Data will be extracted from the participant's electronic medical record and stored in a secure, password-protected file on the secure computer network owned by the health service. Patient-reported outcomes data will be collected and managed securely using Research Electronic Data
Capture hosted at the University of Sydney. ${ }^{47}$ Researchers external to the clinical setting will only be able to access de-identified data, and data will be aggregated and anonymised during analysis and publication to render individual patient identification impossible. Access to deidentified, individual participant data after the completion of the study will be considered on a case-by-case basis in consultation with local researchers and the local human ethics committee. Email contact with the lead author or the relevant ethics committee WSLHD-ResearchOfffice@ health.nsw.gov.au is recommended.

\section{Author affiliations}

${ }^{1}$ Physiotherapy Department, Blacktown Mt Druitt Hospital, Western Sydney Local Health District, Blacktown, New South Wales, Australia

${ }^{2}$ Discipline of Physiotherapy, Sydney School of Health Sciences, Faculty of Medicine and Health, University of Sydney, Sydney, New South Wales, Australia

${ }^{3}$ Department of Health Psychology, Institute of Psychiatry, Psychology \&

Neuroscience (IoPPN), King's College London, London, United Kingdom

${ }^{4}$ Department of Population Health Sciences, School of Population Health \& Environmental Sciences, Faculty of Life Sciences \& Medicine, King's College London, London, United Kingdom

${ }^{5}$ Renal Supportive Care, Nepean Blue Mountains Local Health District, Penrith, New South Wales, Australia

${ }^{6}$ Orthopaedic Department, Blacktown Mt Druitt Hospital, Western Sydney Local Health District, Blacktown, New South Wales, Australia

${ }^{7}$ South Western Sydney Local Health District, Liverpool, New South Wales, Australia ${ }^{8}$ Ingham Institute of Applied Medical Research, Liverpool, New South Wales, Australia

\section{Twitter Marie K March @Physio_Marie}

Acknowledgements The authors acknowledge David Espinosa, Biostatistician, University of Sydney Clinical Trials Centre, for assisting with statistical considerations during study design and Dr Duncan Critchley, Senior Lecturer, Department of Physiotherapy, King's College London, for being involved in developing the KOMPACT intervention described in the study protocol.

Contributors MKM, SD, AH, EG: conceived the study and study design. SV and BT: provided expert guidance in study design relevant to their clinical experience. MKM: drafted the study protocol. All authors were involved in editing and revising the protocol for critically important context; approved the final version.

Funding This work was supported by the University of Sydney and Western Sydney Local Health District via two small grants: Kickstarter Allied Health Research Grant 2017 and Sydney Musculoskeletal Bone and Joint Health Alliance Enabling Research Grant 2017.

Competing interests None declared.

Patient consent for publication Not required.

Provenance and peer review Not commissioned; externally peer reviewed.

Open access This is an open access article distributed in accordance with the Creative Commons Attribution Non Commercial (CC BY-NC 4.0) license, which permits others to distribute, remix, adapt, build upon this work non-commercially, and license their derivative works on different terms, provided the original work is properly cited, appropriate credit is given, any changes made indicated, and the use is non-commercial. See: http://creativecommons.org/licenses/by-nc/4.0/.

ORCID iD

Marie K March http://orcid.org/0000-0003-3444-0795

\section{REFERENCES}

1 Kurtz SM, Ong KL, Lau E, et al. International survey of primary and revision total knee replacement. Int Orthop 2011;35:1783-9.

2 Australian Orthopaedic Association. Australian orthopaedic association national joint replacement registry, hip, knee and shoulder arthroplasty: 2018 annual report. Adelaide, Australia, 2018. 
3 Independent Hospital Pricing Authority. Independent Hospital pricing authority annual report 2013-14. Sydney: IHPA, 2014.

4 Ackerman IN, Bohensky MA, Zomer E, et al. The projected burden of primary total knee and hip replacement for osteoarthritis in Australia to the year 2030. BMC Musculoskelet Disord 2019;20:90.

5 Halawi MJ, Chiu D, Gronbeck C, et al. Psychological distress independently predicts prolonged hospitalization after primary total hip and knee arthroplasty. J Arthroplasty 2019;34:1598-601.

6 March MK, Harmer AR, Dennis S. Does psychological health influence Hospital length of stay following total knee arthroplasty? A systematic review. Arch Phys Med Rehabil 2018;99:2583-94.

7 Khatib Y, Madan A, Naylor JM, et al. Do psychological factors predict poor outcome in patients undergoing TKA? A systematic review. Clin Orthop Relat Res 2015;473:2630-8.

8 Clement ND, MacDonald D, Burnett R. Primary total knee replacement in patients with mental disability improves their mental health and knee function: a prospective study. Bone Joint $J$ 2013;95B:360-6.

9 Wallis JA, Taylor NF. Pre-operative interventions (non-surgical and non-pharmacological) for patients with hip or knee osteoarthritis awaiting joint replacement surgery--a systematic review and metaanalysis. Osteoarthritis Cartilage 2011;19:1381-95.

10 McDonald S, Page MJ, Beringer K, et al. Preoperative education for hip or knee replacement. Cochrane Database Syst Rev 2014;31.

11 das Nair R, Mhizha-Murira JR, Anderson P, et al. Home-Based pre-surgical psychological intervention for knee osteoarthritis (HAPPiKNEES): a feasibility randomized controlled trial. Clin Rehabil 2018;32:777-89.

12 Riddle DL, Keefe FJ, Nay WT, et al. Pain coping skills training for patients with elevated pain catastrophizing who are scheduled for knee arthroplasty: a quasi-experimental study. Arch Phys Med Rehabil 2011;92:859-65.

13 Keefe FJ, Main CJ, George SZ. Advancing Psychologically informed practice for patients with persistent musculoskeletal pain: promise, pitfalls, and solutions. Phys Ther 2018;98:398-407.

14 Guerrero Silva VA, Maujean A, Campbell L, et al. A systematic review and meta-analysis of the effectiveness of psychological interventions delivered by physiotherapists on pain, disability and psychological outcomes in musculoskeletal pain conditions. Clin J Pain 2018;34:1-857.

15 Bryant C, Lewis P, Bennell KL, et al. Can physical therapists deliver a pain coping skills program? an examination of training processes and outcomes. Phys Ther 2014;94:1443-54.

16 Wetherell JL, Petkus AJ, Alonso-Fernandez M, et al. Age moderates response to acceptance and commitment therapy vs. cognitive behavioral therapy for chronic pain. Int J Geriatr Psychiatry 2016;31:302-8.

17 Levin ME, MacLane C, Daflos S, et al. Examining psychological inflexibility as a transdiagnostic process across psychological disorders. J Contextual Behav Sci 2014;3:155-63.

18 Bannuru RR, Osani MC, Vaysbrot EE, et al. OARSI guidelines for the non-surgical management of knee, hip, and polyarticular osteoarthritis. Osteoarthritis Cartilage 2019;27:1578-89.

19 ACSQHC. Australian Commission on safety and quality in health care, osteoarthritis of the Knee- clinical care standard. Sydney, 2017.

20 Godfrey E, Wileman V, Galea Holmes M, et al. Physical therapy informed by acceptance and commitment therapy (PACT) versus usual care physical therapy for adults with chronic low back pain: a randomized controlled trial. J Pain 2019. doi:10.1016/j. jpain.2019.05.012

21 Barker KL, Heelas L, Toye F. Introducing acceptance and commitment therapy to a physiotherapy-led pain rehabilitation programme: an action research study. Br J Pain 2016;10:22-8.

22 Hayes SC, Luoma JB, Bond FW, et al. Acceptance and commitment therapy: model, processes and outcomes. Behav Res Ther 2006:44:1-25.

23 Ferreira $\mathrm{PH}$, Ferreira ML, Maher CG, et al. The therapeutic alliance between clinicians and patients predicts outcome in chronic low back pain. Phys Ther 2013;93:470-8.

24 Kinney M, Seider J, Beaty AF, et al. The impact of therapeutic alliance in physical therapy for chronic musculoskeletal pain: a systematic review of the literature. Physiother Theory Pract 2018;6:1-13.

25 Whitehead AL, Julious SA, Cooper CL, et al. Estimating the sample size for a pilot randomised trial to minimise the overall trial sample size for the external pilot and main trial for a continuous outcome variable. Stat Methods Med Res 2016;25:1057-73.

26 Tam E, Gandesbery BT, Young L, et al. Graphical Instructions for administration and scoring the Mini-Cog: results of a randomized clinical trial. J Am Geriatr Soc 2018;66:987-91.

27 Gloster AT, Rhoades HM, Novy D, et al. Psychometric properties of the depression anxiety and stress Scale-21 in older primary care patients. J Affect Disord 2008;110:248-59.

28 Yohannes AM, Dryden S, Hanania NA. Validity and Responsiveness of the Depression Anxiety Stress Scales-21 (DASS-21) in COPD. Chest 2019;155): :1166-77.

29 Michie S, Wood CE, Johnston M, et al. Behaviour change techniques: the development and evaluation of a taxonomic method for reporting and describing behaviour change interventions (a suite of five studies involving consensus methods, randomised controlled trials and analysis of qualitative data). Health Technol Assess 2015;19): :1-188.

30 Psychwire. Act for beginners. I'm learning act, 2020. Available: https://psychwire.com/harris/act-beginners

31 Borkovec TD, Nau SD. Credibility of analogue therapy rationales. $J$ Behav Ther Exp Psychiatry 1972;3:257-60.

32 Smith BW, Dalen J, Wiggins K, et al. The brief resilience scale: assessing the ability to bounce back. Int J Behav Med 2008:15:194-200.

33 McCracken LM, Chilcot J, Norton S. Further development in the assessment of psychological flexibility: a shortened committed action questionnaire (CAQ-8). Eur J Pain 2015;19:677-85.

34 Fish RA, Hogan MJ, Morrison TG, et al. Willing and able: a closer look at pain willingness and activity engagement on the chronic pain acceptance questionnaire (CPAQ-8). J Pain 2013;14:233-45.

35 Nicholas MK, McGuire BE, Asghari A. A 2-item short form of the pain self-efficacy questionnaire: development and psychometric evaluation of PSEQ-2. J Pain 2015;16:153-63.

36 Beard DJ, Harris K, Dawson J, et al. Meaningful changes for the Oxford hip and knee scores after joint replacement surgery. J Clin Epidemiol 2015;68:73-9.

37 Hawker GA, Mian S, Kendzerska T, et al. Measures of adult pain: visual analog scale for pain (vas pain), numeric rating scale for pain (NRS pain), McGill pain questionnaire (MPQ), short-form McGill pain questionnaire (SF-MPQ), chronic pain grade scale (CpGs), short Form-36 bodily pain scale (SF-36 BPs), and measure of intermittent and constant osteoarthritis pain (ICOAP). Arthritis Care Res 2011;63:S240-52.

38 Hurtig-Wennlöf A, Hagströmer M, Olsson LA. The International physical activity questionnaire modified for the elderly: aspects of validity and feasibility. Public Health Nutr 2010;13:1847-54.

39 Conner-Spady BL, Marshall DA, Bohm E, et al. Reliability and validity of the EQ-5D-5L compared to the EQ-5D-3L in patients with osteoarthritis referred for hip and knee replacement. Qual Life Res 2015;24:1775-84.

40 Ko V, Naylor JM, Harris IA, et al. The six-minute walk test is an excellent predictor of functional ambulation after total knee arthroplasty. BMC Musculoskelet Disord 2013:14:145.

41 Dobson F, Hinman RS, Roos EM, et al. OARSI recommended Performance-based tests to assess physical function in people diagnosed with hip or knee osteoarthritis. Osteoarthritis Cartilage 2013;21:1042-52.

42 Unver B, Kalkan S, Yuksel E, et al. Reliability of the 50-foot walk test and $30-\mathrm{sec}$ chair stand test in total knee arthroplasty. Acta Ortop Bras 2015;23:184-7.

43 Braun V, Clarke V. Using thematic analysis in psychology. Qual Res Psychol 2006;3:77-101.

44 Chan A-W, Tetzlaff JM, Altman DG, et al. Spirit 2013 statement: defining standard protocol items for clinical trials. Ann Intern Med 2013;158:200-7.

45 Hoffmann TC, Glasziou PP, Boutron I, et al. Better reporting of interventions: template for intervention description and replication (TIDieR) checklist and guide. BMJ 2014;348:g1687.

46 Eldridge SM, Chan CL, Campbell MJ, et al. Consort 2010 statement: extension to randomised pilot and feasibility trials. BMJ 2016;355:i5239.

47 Harris PA, Taylor R, Thielke R, et al. Research electronic data capture (REDCap)--a metadata-driven methodology and workflow process for providing translational research informatics support. J Biomed Inform 2009;42:377-81. 